\title{
Nonlinear periodic solutions for isothermal magnetostatic atmospheres
}

\author{
A. H. Khater ${ }^{1,2}$, D. K. Callebaut ${ }^{2}$ and E. S. Kamel ${ }^{3}$ \\ ${ }^{1}$ Department of Mathematics, Faculty of Science, Beni-Suef University, Beni-Suef, Egypt \\ email: khater_ah@hotmail.com \\ ${ }^{2}$ Departement Natuurkunde, CGB, University of Antwerp (UA), B-2020 Antwerp, Belgium \\ email: dirk.callebaut@ua.ac.be \\ ${ }^{3}$ Mathematics Department, Faculty of Science, Fayoum University, Fayoum,Egypt
}

\begin{abstract}
Magnetohydrodynamic equilibria for a plasma in a gravitational field are investigated analytically. For equilibria with one ignorable spatial coordinate, the equations reduce to a single nonlinear elliptic partial differential equation for the magnetic potential A, known as the Grad-Shafranov equation. Specifying the arbitrary functions in the latter equation, one gets a nonlinear elliptic partial differential equation (the sinh Poisson equation). Analytical solutions of this equation are obtained for the case of an isothermal atmosphere in a uniform gravitational field. The solutions are obtained by using the tanh method, and are adequate for describing parallel filaments of diffuse, magnetized plasma suspended horizontally in equilibrium in a uniform gravitational field.
\end{abstract}

Keywords. Hydromagnetics, Stars, Sun, Magnetic fields

\section{Introduction}

The equations of magnetohydrostatic (MHS) equilibria have been used extensively to model solar magnetic structures, see Khater et al. (1998, 2002a) for details and review. The force balance in these models consists of a balance between the pressure gradient force, the $j \wedge \underline{B}$ force ( $j$, electric current density, $\underline{B}$ magnetic induction), and the gravitational force. The temperature distribution in the atmosphere is in general determined from the energy transport equation. However, in many models, the temperature distribution is specified a priori, and direct reference to the energy equation is eliminated. The remaining equations for the system are an equation of state for the gas (e.g., the dependence of the gas pressure on density and temperature) and the steady state Maxwell equations. In solar physics the MHS equations have been used to model diverse phenomena, such as the slow evolution stage of solar flares, or the MHS support of prominences (Low et al., 1983; Khater et al., 1997; 1998).

In this paper, we present a class of periodic solutions for the sinh Poisson equation modeling an isothermal MHS atmosphere by applying the tanh method (Khater et al. $2002 \mathrm{~b}$ ), for the two-dimensional boundary value problem in the solar surface $x \geqslant 0$ but only for a bounded domain (Heyvaerts et al., 1982; Low et al., 1983; Amari and Aly, 1989). We consider an isothermal atmosphere with one ignorable coordinate $x$ of a rectangular Cartesian coordinate system $O x y z$ in which the gravitational force is directed in the negative $z$ direction. We study the solutions with $j_{x}=-\left(\lambda A_{0} / 4 h\right) \sinh \tilde{A} e^{-z / h}$. For this current the components of the force balance equation perpendicular to both $\underline{B}$ and $e_{x}\left(e_{x}\right.$ is the unit vector along the $x$-axis $)$ reduces to a sinh Poisson equation. Through exact solutions obtained in this way, interesting physical properties can be deduced in qualitative form. 
The paper is organized as follows: In section 2, the basic equations and the formulation of the nonlinear problem, which reduces to the sinh Poisson equation, are illustrated and reviewed. In section 3 , the tanh solution method for the partial differential equations is briefly discussed together with exact analytical periodic solutions for plasma models. Finally, the analytical results, together with graphical representations are given and discussed in section 4 .

\section{Basic equations}

The equations used to describe a MHS atmosphere consists of the force balance equation

$$
\underline{j} \wedge \underline{B}-\nabla P-\rho \nabla \Phi=0,
$$

coupled with Maxwell's equations

$$
\underline{j}=\nabla \wedge \underline{B}, \quad \nabla \cdot \underline{B}=0,
$$

where $P, \rho$ and $\Phi$ are the gas pressure, the mass density and the gravitational potential, respectively. We assume the temperature is uniform in space and the plasma is an ideal gas with equation of state

$$
P=\rho R_{0} T_{0},
$$

where $R_{0}$ is the gas constant and $T_{0}$ is the temperature.

Consider a system of Cartesian coordinates $O x y z$, in which $x$ is an ignorable coordinate and $z$ measures the height above a reference surface taken as the $x y$-plane, then the magnetic induction $\underline{B}$ may be written as

$$
\underline{B}=\nabla A \wedge e_{x}+B_{x} e_{x}\left(B_{x}, \frac{\partial A}{\partial z}, \frac{\partial A}{\partial y}\right)
$$

where $A(y, z)$ and $B_{x}(y, z)$ are the magnetic flux function and $x$-component of $\underline{B}$, respectively. Note that the form (2.4) for $\underline{B}$ ensures that $\nabla \cdot \underline{B}=0$. Since $\underline{B} \cdot \nabla A=0$, $A(y, z)$ is constant along the magnetic lines of force. We restrict our attention to isothermal atmospheres in a uniform gravitational field $(\Phi=g z)$, in which $B_{x}=0$ and using the ideal gas law (2.3) to relate the pressure and density to the uniform temperature $T_{0}$ of the atmosphere. Equation (2.1) then requires that the pressure and density have the form (Webb, 1988)

$$
P(y, z)=P(A) e^{-z / h}, \quad \rho(y, z)=\frac{1}{g h} P(A) e^{-z / h},
$$

where $h=R_{0} T_{0} / g$ is the (constant) scale height, and $P(A)$ is an arbitrary function of one variable to describe the variation of pressure across the magnetic lines of force at some constant height. Substituting (2.2a), (2.4)-(2.5b) into (2.1), one gets (Low et al., 1983; Khater et al., 1998)

$$
\nabla^{2} A+f(A) e^{-z / h}=0, \quad f(A)=\frac{d P}{d A} .
$$

Subject to suitable boundary conditions on $A$, equation (2.6b) may be solved for $A$ in a given domain if the functional form $P(A)$ is prescribed in some suitable manner (Dungey, 1953). 
The term $f(A)$ is, in general, nonlinear in $A$ raising nontrivial questions of existence, uniqueness, and regularity of solutions to boundary value problems based on (2.6a). Rigorous and general mathematical results on these questions for equation (2.6a) in the nonlinear regime have been obtained and discussed by Amari and Aly (1988; 1989) and Heyvaerts et al. (1982). The absence of a regular solution may be interpreted to imply that electric current sheets may be unavoidable

Equation (2.6b) gives

$$
P(A)=P_{0}+\int f(A) d A .
$$

Substituting equation (2.7) into equations (2.5a) and (2.5b), we get

$$
P(y, z)=\left(P_{0}+\int f(A) d A\right) e^{-z / h}, \quad \rho(x, z)=\frac{1}{g h}\left(P_{0}+\int f(A) d A\right) e^{-z / h}
$$

where $P_{0}$ is constant. Take the conformal transformation

$$
x_{1}+i x_{2}=e^{-z / l} e^{i y / l}
$$

(e.g. Lerche and Low, 1982), equation (2.6a) reduces to

$$
\frac{\partial^{2} A}{\partial x_{1}^{2}}+\frac{\partial^{2} A}{\partial x_{2}^{2}}+l^{2} f(A) e^{\left(\frac{2}{l}-\frac{1}{h}\right) z}=0
$$

Let us identify the period $l$ by:

$$
2 / l=2 / L+1 / h
$$

Note that using the transformation (13), and taking $l>0$, we have transformed the region $0 \leqslant y \leqslant 2 \pi l, 0 \leqslant z \leqslant \infty$ into the entire $x_{1}-x_{2}$ plane with origin $x_{1}=x_{2}=0$ corresponding to $z \rightarrow \infty$ and the region $x_{1}^{2}+x_{2}^{2} \rightarrow 1$ corresponding to $z \rightarrow 0$. Note that in the limit of an infinite period $l$ as $l \rightarrow \infty$, equation (2.11) implies $2 / L=-1 / h$.

Let us assume that $f(A)$ has the form (Khater et al., 1988; 1997; 2002a):

$$
f(A)=-\frac{\lambda^{2}}{4}\left(\frac{A_{0}}{h}\right) \sinh (\tilde{A}), \quad \tilde{A}=A /\left(h A_{0}\right),
$$

is a dimensionless form of $A$, where $\lambda$ is a dimensionless constant.

Equations (2.5a), (2.7) and (2.11) give

$$
P(y, z)=\left(P_{0}-\frac{\lambda^{2} A_{0}^{2}}{4 \mu} \cosh \tilde{A}\right) e^{-z / h}
$$

The corresponding form of equation (2.10) is given by using equations (2.10) and (2.11) as

where $l=2 h$.

$$
\frac{\partial^{2} \tilde{A}}{\partial x_{1}^{2}}+\frac{\partial^{2} \tilde{A}}{\partial x_{2}^{2}}=\lambda^{2} \sinh (\tilde{A})
$$

Equation (2.13) is the well known sinh-Poisson equation. To solve (2.14), we are looking for the solution where $A$ is periodic in $y$ with period $2 \pi l$ (Low et al., 1983; Khater et al., 1998)

$$
A(y+2 \pi l, z)=A(y, z)
$$

which corresponds to an array of plasma condensations or current filaments that are arranged periodically in the $y$-direction. These condensations have to be of finite extent vertically. Hence in the far region $z \rightarrow \pm \infty$, the field is required to be horizontal and 
uniform. The following boundary conditions apply

$$
\lim _{z \rightarrow \pm \infty} \underline{B}_{ \pm}=A_{0}\left(\frac{1}{l}-\frac{1}{2 h}\right) \hat{y}=B_{ \pm} \hat{y}
$$

where $B_{+}$is the constant field strength. Equation (2.14) is a nonlinear elliptic partial differential equation (Courant and Hilbert, 1963; Birn et al., 1978; Low, 1982); one can not take for granted that boundary conditions (2.15) and (2.16) admit a solution and, where a solution exists, that it is unique. Let us set

$$
A / A_{0}=z / L+u(y, z)
$$

where $L$ is a constant.

To solve equations (2.14) analytically, we use the tanh method which we discuss in the following section.

\section{Solution method and exact analytical solution for plasma condensations models}

Various forms of the tanh method have been introduced. First a power series in tanh was used as ansatz to obtain analytical solutions of traveling wave type of certain nonlinear evolution equations. In the next stage, Malfliet (1991) made a first improvement of the method through the change of the independent variable $\zeta=c(x-\nu t)$, typical for traveling waves, and the use of the hyperbolic function, $\tanh (\zeta)$; itself as a new variable. As a result, the involved algebra was considerably reduced compared to that in the first stage. Later on, this technique was refined by incorporating the boundary conditions in the series expansion and by determining the velocity through asymptotics (Malfliet 1992; 1993). The method was extended to higher dimensions (Khater et al. 2002b). Now, we explore the method to solve equation (2.14), then we use it for the plasma condensation model. Consider the traveling wave solution

$$
\tilde{A}\left(x_{1}, x_{2}\right)=U(\zeta), \quad \zeta=c\left(x_{1}-\nu x_{2}\right),
$$

then equation (2.14) is reduced to the ordinary differential equation

$$
\frac{d^{2} U}{d \zeta^{2}}=\frac{\lambda^{2} \sinh (U)}{c^{2}\left(1+\nu^{2}\right)}, \quad U(\zeta) \rightarrow 0, \quad \frac{d U}{d \zeta} \quad \text { and } \quad \frac{d^{2} U}{d \zeta^{2}} \rightarrow 0 \quad \text { as } \quad \zeta \rightarrow \infty .
$$

Multiplying both sides of equation (3.2) by $\frac{d U}{d \zeta}$ and integrating it, we get

$$
c \sqrt{1+\nu^{2}} \frac{d H}{d \zeta}-\frac{\lambda}{2}\left(H^{2}-1\right)=0, \quad H(\zeta) \rightarrow 1, \quad \frac{d H}{d \zeta} \rightarrow 0 \quad a s \zeta \rightarrow \infty,
$$

where the integration constant is taken to be zero and

$$
H=e^{U / 2} \text {. }
$$

Put

$$
Z=\tanh (\zeta), \quad H(\zeta)=S(Z)=\sum_{n=0}^{M} b_{n} Z^{n}
$$

Equations (3.3) and (3.4) yield

$$
c \sqrt{1+\nu^{2}}\left(1-Z^{2}\right) \frac{d S}{d Z}-(\lambda / 2)\left(S^{2}-1\right)=0 .
$$


The balance between the linear term and the nonlinear one in the equation (3.6) gives $M=1$, and the solution of it is written as:

$$
S(Z)=b_{0}+b_{1} Z
$$

Substituting (3.7) into (3.6), and comparing the coefficients of each power of $\mathrm{Z}$ in both sides we can get

$$
b_{0}=0, \quad b_{1}=1 \quad \text { and } \quad c=-\frac{\lambda}{2 \sqrt{1+\nu^{2}}} .
$$

Thus the solution of equation (2.14) can be written as

$$
A / A_{0}=-4 h \operatorname{coth}^{-1}\left[\exp \left(\frac{-\lambda}{\sqrt{\left(1+\nu^{2}\right)}} e^{-z / 2 h}(\cos y / 2 h-\nu \sin y / 2 h)\right)\right],
$$

where equations $(2.9),(2.15)$ have been used and $l=2 h$.

The associated magnetic induction and pressure are given by using equations $(2.4),(2.15)$, (2.16) and (3.8)

$$
\begin{aligned}
& \underline{B} / A_{0}=\left\{0,-\frac{\lambda}{\sqrt{\left(1+\nu^{2}\right)}} e^{-z / 2 h}(\cos (y / 2 h)-\nu \sin (y / 2 h)) \operatorname{cosech}\left[\frac{\lambda}{\sqrt{\left(1+\nu^{2}\right)}} e^{-z / 2 h}\right.\right. \\
&(\cos (y / 2 h)-\nu \sin (y / 2 h))], \frac{\lambda}{\sqrt{\left(1+\nu^{2}\right)}} e^{-z / 2 h}(\sin (y / 2 h)+\nu \cos (y / 2 h)) \\
&\left.\operatorname{cosech}\left[\frac{\lambda}{\sqrt{\left(1+\nu^{2}\right)}} e^{-z / 2 h}(\cos (y / 2 h)-\nu \sin (y / 2 h))\right]\right\} . \\
& P=P_{0} e^{-z / l}+\frac{\lambda^{2} A_{0}^{2}}{4 \mu} e^{-z / h}\left[1-2 \operatorname{coth}^{2}\left(\frac{\lambda}{\sqrt{\left(1+\nu^{2}\right)}} e^{-z / 2 h}(\cos y / 2 h-\nu \sin y / 2 h)\right)\right]
\end{aligned}
$$

The magnetic and plasma pressures are given by

$$
\begin{gathered}
P=\frac{\lambda^{2} A_{0}^{2}}{2 \mu} e^{-z / h} \operatorname{cosech}^{2}\left[\frac{\lambda}{\sqrt{\left(1+\nu^{2}\right)}} e^{-z / 2 h}(\cos y / 2 h-\nu \sin y / 2 h)\right], \\
P=\frac{\lambda^{2} A_{0}^{2}}{4 \mu} e^{-z / h}\left\{1-2 \operatorname{coth}^{2}\left[\frac{\lambda}{\sqrt{\left(1+\nu^{2}\right)}} e^{-z / 2 h}(\cos y / 2 h-\nu \sin y / 2 h)\right]\right\},
\end{gathered}
$$

The magnetic and plasma pressures are displayed in figures $[1(a, b)-2(a, b)]$ in the surface and plane graphics (in the plane graphic $y=$ constant $=\pi / 3$ and $z=$ constant $=\ln 2$ ), where $A_{0}^{2}=2 \mu, \lambda=1.4, h=1$, and $\nu=1$. In Figure $3(a, b)$, density enhancement is displayed relative to the associated magnetic field distributions, where $\beta=\ln \left[\operatorname{coth}\left(\frac{-A / A_{0}}{4 h}\right)\right]$ and $\gamma=\frac{2 \mu}{\lambda^{2} A_{0}^{2}} \sqrt{P-P_{0} e^{-z / h}}$.

For the magnetic surfaces (contours of A in which A is constant along any field line) and density enhancement (in which $P-P_{0} e^{-z / h}$ is a constant) we use equations (3.9) and (3.13) to obtain

$$
\begin{gathered}
\cos Y-\nu \sin Y=-\frac{\sqrt{1+\nu^{2}}}{\lambda} \beta e^{Z} \\
\cos Y-\nu \sin Y=\frac{\sqrt{1+\nu^{2}}}{\lambda} e^{Z} \operatorname{coth}^{-1} \sqrt{\frac{1-\gamma e^{2 Z}}{2}}
\end{gathered}
$$


(a)

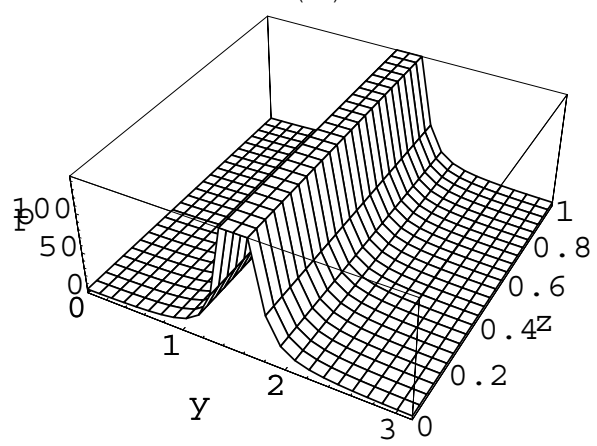

(b)

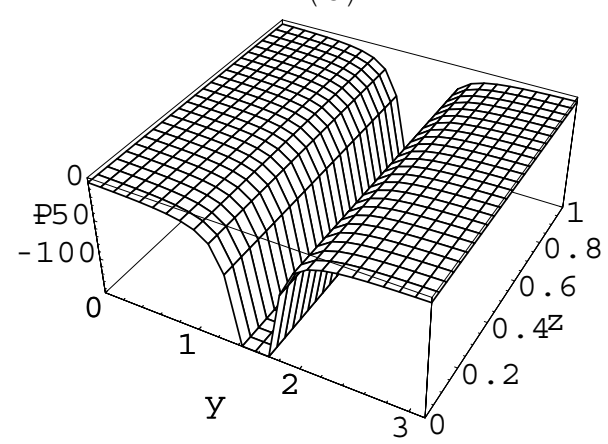

Figure 1. Fig. 1(a): The magnetic pressure corresponding to the solution (3.11) of the sinh Poisson equation (2.14) for $0 \leqslant z \leqslant 1$ and $0 \leqslant y \leqslant \pi$. Fig. 1(b): The plasma pressure (pressure enhancement) corresponding to the solution (3.12) of the sinh Poisson equation (2.14) for $0 \leqslant z \leqslant 1$ and $0 \leqslant y \leqslant \pi$.
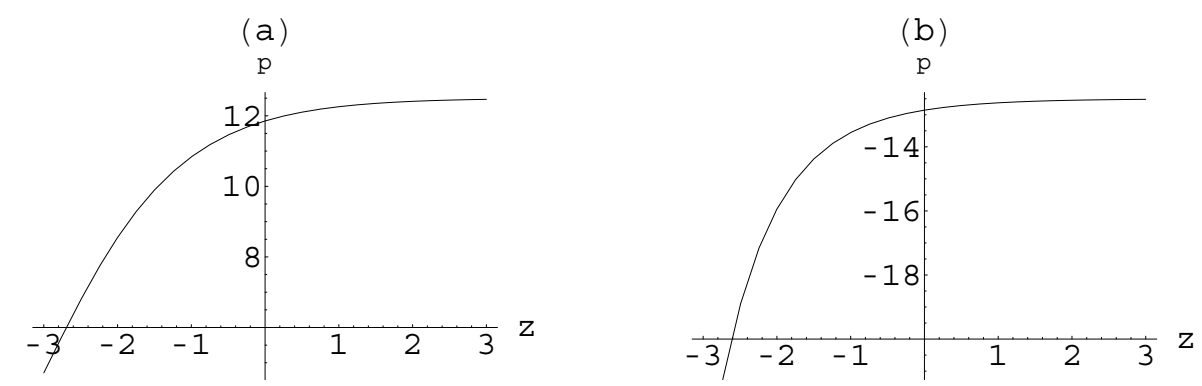

Figure 2. Fig. 2(a): The magnetic pressure corresponding to the solution (3.11) of the sinh Poisson equation (2.14) for $(y<\pi / 3)$. Fig. 2(b): The plasma pressure (pressure enhancement) corresponding to the solution (3.12) of the sinh Poisson equation $(2.14)$ for $(y<\pi / 3)$.

(a)

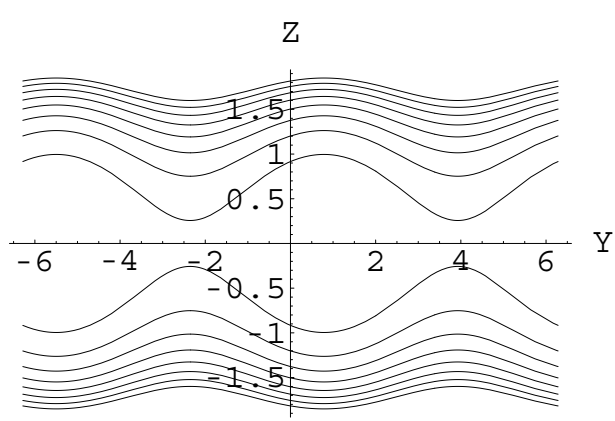

(b)

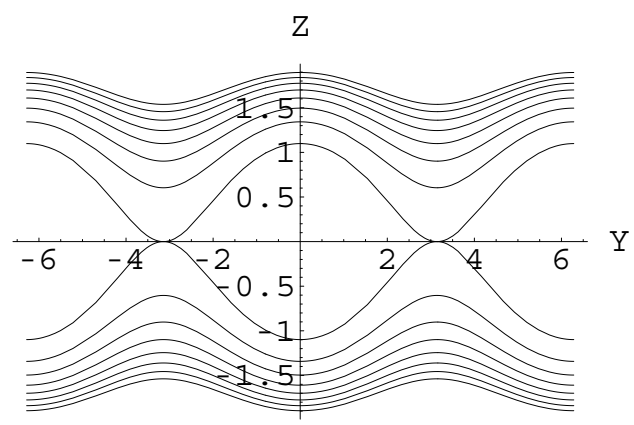

Figure 3. Fig. 3(a): The magnetic field lines (contours of A) corresponding to the solution (3.11) of the sinh Poisson equation (2.14) for $\beta=\gamma_{3}= \pm 0.1, \pm 0.2, \pm 0.3, \pm 0.4, \pm 0.5, \pm 0.6, \pm 0.7, \pm 0.8, \pm 0.9$ and $-2 \pi \leqslant Y \leqslant 2 \pi$. Fig. 3(b): The associated density enhancement corresponding to the solution (3.12) of the sinh Poisson equation (2.14) for $\gamma= \pm 0.1, \pm 0.2, \pm 0.3, \pm 0.4, \pm 0.5, \pm 0.6, \pm 0.7, \pm 0.8, \pm 0.9$ and $-2 \pi \leqslant Y \leqslant 2 \pi$. 
where

$$
\beta=\ln \left[\operatorname{coth}\left(\frac{-A / A_{0}}{4 h}\right)\right], \quad \gamma=\frac{2 \mu}{\lambda^{2} A_{0}^{2}} \sqrt{P-P_{0} e^{-z / h}}, \quad Y=y / 2 h \quad \text { and } \quad Z=z / 2 h .
$$

In Figures 1-3(a,b) density enhancement is displayed relative to the associated magnetic field distributions, where $A_{0}^{2}=2 \mu, h=1, \nu=1$ and $\lambda=1,4$.

\section{Summary and discussion}

In this paper we have investigated isothermal magnetostatic atmospheric models with one ignorable coordinate $x$ of a Cartesian coordinate system $x y z$ in which the distributed current is $j_{x} \sim-\lambda \sinh \left(A / h A_{0}\right) e^{-z / h}$, where $h$ is the gravitational scale height in a constant gravity field, $A_{0}$ is a characteristic value of the magnetic induction, $\alpha$ and $\lambda$ determine the magnitude of the distributed current. The underlying elliptic equation governing the force balance perpendicular to both $\underline{B}$ and $e_{x}$ reduced to the sinh Poisson equation. The main interest of this paper is to obtain classes of nonlinear magnetostatic solutions that are obtained analytically by exploring the tanh method, namely, the solutions corresponding to the particular choice of the pressure profile, given in terms of the magnetic flux function $A$ by equation (2.10).

Our results represent the solutions of the sinh Poisson equation. Figures $(1-3)$ display the run of the magnetic pressure and pressure enhancement (plasma pressure). It represents the subcase in which $y=$ constant $=\pi / 3$ and we notice that the magnetic pressure gradient is steeper below the enhancement center $z=0$ than above. This is due to the magnetic pressure which at $z<0$ has to support the local weight as well as to counter the expansive pressure of enhancement. Whereas, in $z>0$, the enhancement pressure acts upward to assist the magnetic pressure in supporting the local weight.

The figures represent the magnetic field lines. In all subcases the density enhancement is a clump that rests on locally depressed field lines, its weight being supported by an upward Lorentz force. The density enhancement is maximum where the magnetic field lines are most densely packed and depressed. The figures also illustrate the relationship between the configuration of the supporting magnetic field in the neighborhood of the density enhancement and the field configuration in the far region $z \rightarrow \pm \infty$.

\section{References}

Amari, T. \& Aly, J.J. 1988, in : Proc. IAU Colloquium No 104-Solar and Stellar Flares. Haish B.M., Rodono M.(eds) Kluwer, Dordrecht.

Amari, T. \& Aly, J.J. 1989, Astron. Astophys. 208, 361

Birn, J., Goldstein, H. \& Schindler, K. 1978 Solar Phys. 57, 81

Courant, R. \& Hilbert, D. 1963, Methods of Mathematical Physics II (Interscience, New York), Chap.IV.

Heyvaerts, J., Larsy, J.M., Schatzman, E. \& Witomsky, P. 1982 Astron. Astrophys. 111, 104

Khater, A.H., Callebaut, D.K. \& Ibrahim, R.S. 1997, Phys. of Plasmas. 4, 2853

Khater, A.H., Callebaut, D.K. \& Kamel, E.S. 1998, Solar Phys. 178, 285

Khater, A.H., El-Attary, M.A., El-Sabbagh, M.F. \& Callebaut, D.K. 1988, Astrophy. Sp. Sci. $149,217$.

Khater, A.H., Ibrahim, R.S., Shamardan, A.B. \& Callebaut, D.K. (1997), IMA J. Applied Math. 58,51

Khater, A.H., Callebaut, D.K., Malfliet, W. \& Kamel, E.S. 2002a, J. Comp. Appl. Math. 140, 435

Khater, A.H., Malfliet, W., Callebaut, D.K. \& Kamel, E.S. 2002b, J. Comp. Appl. Math. 140, 469 
Lerche, I. \& Low, B.C. 1980, Solar Phys. 67, 229

Lerche, I. \& Low, B.C. 1982, Physica. 4D, 293

Low, B.C., Hundhausen, A.I. \& Zwibel, E.G. 1983, Phys. Fluids 26, 2731

Malfliet, W. 1991, J. Phys. A Math. Gen. 27, 5499

Webb, G.M. 1988, ApJ 327, 933 